\title{
Tunnel Back Analysis Based on Differential Evolution Using Stress and Displacement
}

\author{
Joon-Sang An, Kyung-Nam Kang (D, Ju-Young Choi, Won-Suh Sung, Vathna Suy, \\ and Ki-Il Song $(\mathbb{D}$
}

Department of Civil Engineering, Inha University, 100 Inha-ro, Nam-gu, Incheon 22212, Republic of Korea

Correspondence should be addressed to Ki-Il Song; ksong@inha.ac.kr

Received 5 September 2019; Accepted 9 January 2020; Published 12 March 2020

Academic Editor: Dimitris Rizos

Copyright (c) 2020 Joon-Sang An et al. This is an open access article distributed under the Creative Commons Attribution License, which permits unrestricted use, distribution, and reproduction in any medium, provided the original work is properly cited.

\begin{abstract}
The stability of tunnels has mainly been evaluated based on displacement. Because displacement due to the excavation process is significant, back analysis of the structure and ground can be performed easily. Recently, the length of a segment-lined tunnel driven by the mechanized tunneling method is increasing. Because the internal displacement of a segment-lined tunnel is trivial, it is difficult to analyze the stability of segment-lined tunnels using the conventional method. This paper proposes a back analysis method using stress and displacement information for a segment-lined tunnel. A differential evolution algorithm was adopted for tunnel back analysis. Back analysis based on the differential evolution algorithm using stress and displacement was established and performed using the finite difference code, FLAC3D, and built-in FISH language. Detailed flowcharts of back analysis based on DEA using both monitored displacement stresses were also suggested. As a preliminary study, the target variables of the back analysis adopted in this study were the elastic modulus, cohesion, and friction angle of the ground. The back analysis based on the monitored displacement is useful when the displacement is significant due to excavation. However, the conventional displacement-based back analysis is unsuitable for a segment-lined tunnel after construction because of its trivial internal displacement since the average error is greater than $32 \%$ and the evolutionary calculation is finalized due to the maximum iteration criteria. The average error obtained from the proposed back analysis algorithm using both stress and displacement ranged within approximately $6-8 \%$. This also confirms that the proposed back analysis algorithm is suitable for a segment-lined tunnel.
\end{abstract}

\section{Introduction}

The stability of a geoinfrastructure has been evaluated mainly based on displacement (Zhang et al. [1], Zhou et al. [2], and Gao and He [3]). Because the displacement due to the excavation process is significant, the analysis of the structure and ground can be performed easily. This process is generally known as back analysis. In engineering problems, back analysis estimates the design variable of the load and material properties from monitored strain and displacement responses under the boundary conditions of a structure. Back analysis is divided largely into the direct method, inversion method, and statistical methods (Hisataki and Ito [4] and Gioda and Sakurai [5]). Figure 1 presents an overview of the representative back analysis methods.
Various back analysis methods based on displacement have been suggested for a safety assessment during tunnel construction. Vardakos [7] proposed several back analysis methods for the optimal design of the NATM (New Austrian Tunnelling Method) tunnel. Su et al. [8] developed a tunnel back analysis algorithm using the Differential Evolution Algorithm (DEA). Lee and Akutagawa [9] studied back analysis through the displacement measurement results of the NATM tunnel. An ANN (Artificial Neural Network) was constructed using back analysis algorithm techniques and the possible methods for predicting the deformation of the NATM tunnel from preconstruction information were suggested. Vardakos et al. [10] constructed a back analysis algorithm to calculate the tunnel surrounding ground properties by combining the Differential Evolution Algorithm and Genetic Algorithm. Although many researchers 


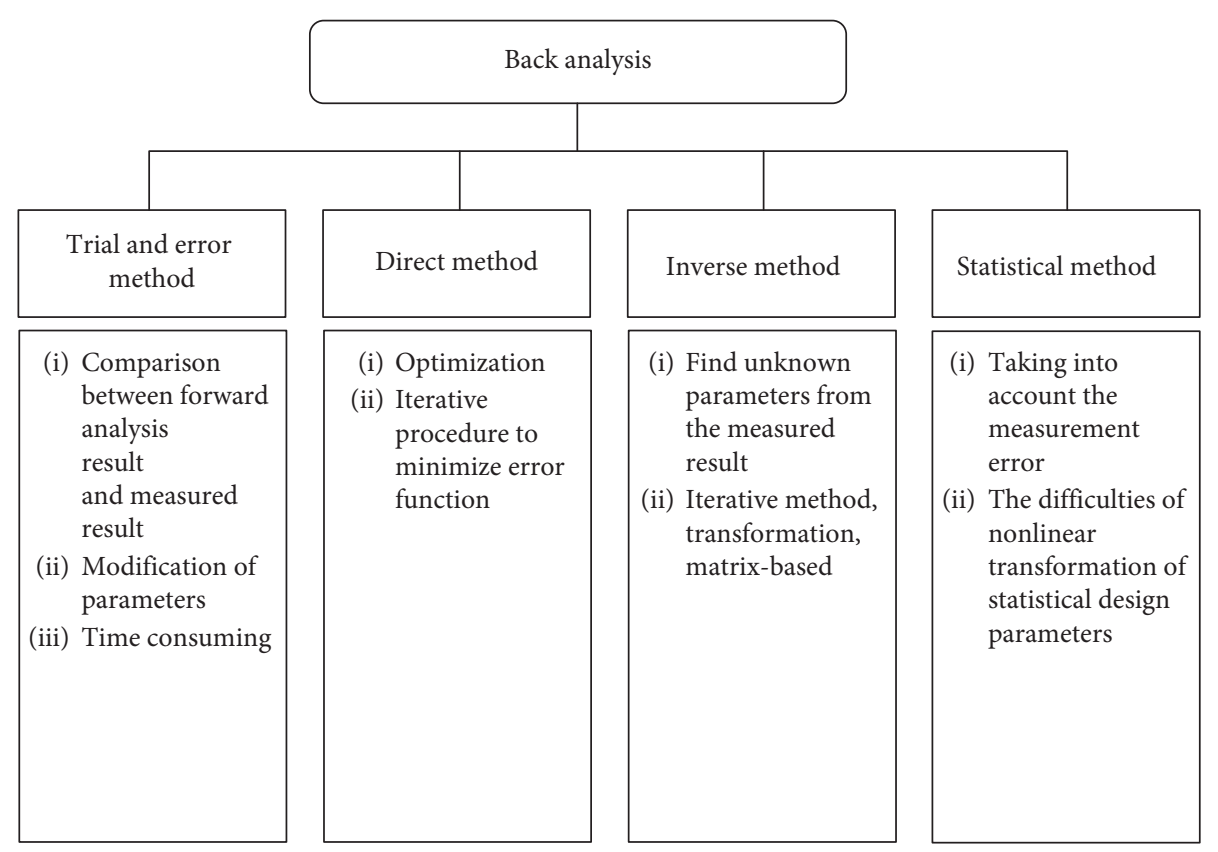

FIgure 1: Classification of back analysis (An and Song [6]).

have performed back analysis to assess the tunnel stability using the monitored displacement, back analysis was available only on a tunnel under construction, where the displacement is significant and an acceptable convergence level can be obtained.

Recently, the length of a segment-lined tunnel driven by a mechanized tunneling method has been increasing. The internal displacement of a segment-lined tunnel is trivial after its construction. In such case, it is difficult to analyze the stability of a segment-lined tunnel using the conventional method based on displacement due to the convergence problem. On the other hand, few back analysis studies have evaluated the stability of a segment-lined tunnel. Therefore, it is important to improve the conventional back analysis scheme for a segment-lined tunnel extending the target variables and observation variables.

This paper proposes the back analysis method using both stress and displacement information for a segment-lined tunnel. The differential evolution algorithm was adopted for tunnel back analysis. The back analysis based on the differential evolution algorithm using both stress and displacement was established and performed using the finite difference code, FLAC3D, and built-in FISH language (Itasca Consulting Group Inc. [11]). The target variables of back analysis adopted in this study were the elastic modulus, cohesion, and friction angle of the ground. The performance of the suggested algorithm was examined under a range of boundary conditions.

\section{Revisit to Differential Evolution Algorithm}

Differential Evolution Analysis (DEA) was developed to optimize the nonlinear and nondifferentiable in the continuous space function (Hwang [12]). DEA is a searching algorithm that uses the target variable vector of the population size. To evaluate the performance of DEA, Vesterstrøm and Thomsen [13] compared DEA with various back analysis algorithms. The performance of DEA, Particle Swarm Optimization (PSO), and Evolution Algorithm (EA) was analyzed with respect to 34 different types of chosen benchmark functions. DEA was found to be more efficient than PSO and EA and it can be used as a strong back analysis algorithm for nonlinear problems. The application of the DEA in locating earthquake hypocenter has been examined by Růžek and Kvasnička [14]. An insight into the use and implementation of DEA algorithms is also given by Reed and Yamaguchi [15]. The use of the DEA in estimation of rock fracture sizes is described by Decker and Mauldon [16]. DEA also has been used for the detection of structural damage in structural engineering field (Seyedpoor et al. [17], Seyedpoor and Montazer [18], and Zhang et al. [1]). Despite being used in various fields with respect to the optimization problem, DEA is not used widely in the geotechnical engineering field.

DEA was initially proposed by Storn and Price [19]; it uses the distance and direction data of the object vector, which belongs to the population. Although the algorithm structure and operation of DEA are simple, the convergence is fast. In addition, DEA has few algorithm-controlling parameters, making it easier to use and suitable for parallel processing. DEA has the same basic operations as EA, which include generating an initial population randomly and extracting the outstanding objects through mutation, crossover, and selection stage. On the other hand, the EA coding process is no longer necessary because DEA takes an object as a vector form. Furthermore, DEA produces a new entity based on the arithmetic operation for each entity. DEA uses operations, such as mutation, crossover, and selection, which are similar to the Genetic Algorithm (GA). The main difference between the two methods is that DEA is dependent of mutations, whereas the GA depends on 
crossover operations (Su et al. [8]). Figure 2 shows the main steps of DEA. In Figure 2, D denotes the dimensional vector. $\mathrm{NP}$ is the population size. $\mathrm{F}$ is the mutation constant with values between 0 and 2 . CR is the crossover constant with a value between 0 and 1 . $G$ denotes generation in the evolution process.

For each target variable vector, $x_{G}=\left[x_{1, G}, x_{2, G}\right.$, $\left.\ldots, x_{\mathrm{NP}, G}\right]$ in a population, a new generation vector can be expressed as equation (1). The new generation vector is called the crossover vector, which is generated from three different randomly selected vectors. Figure 3 presents a twodimensional mutant vector of equation (1) in two-dimensional space. Equation (2) provides information on the crossover stage. The parent vector is crossed with a mutant vector to generate a trial vector, wherein rand means a randomly selected change vector and Irand is a random integer within $[1,2, \ldots, \mathrm{D}]$.

Equation (3) expresses the selection stage. The target vector $\left(x_{i, G+1}\right)$ is compared with the trial vector $\left(u_{i, G+1}\right)$; good object traits are selected for the next generations. Therefore, each trial vector transfers excellent traits to the next generation through a comparison with the parent vector. On the other hand, excellent objects in the population are maintained in the next generation. This process is repeated until a specific exit criterion is reached. In other words, mutation, crossover, and selection continue until a certain stopping criterion is satisfied.

$$
\begin{aligned}
& v_{i, G+1}=x_{r 1, G}+F\left(x_{r 2, G}-x_{r 3, G}\right), \\
& v_{j i, G+1}= \begin{cases}v_{j i, G+1}, & \text { if } \operatorname{rand}_{j} \leq \mathrm{CR} \text { or } j=I_{\text {rand }}, \\
v_{j i, G}, & \text { if } \operatorname{rand}_{j}>\operatorname{CR} \text { or } j \neq I_{\text {rand }},\end{cases} \\
& x_{i, G+1}= \begin{cases}u_{i, G+1}, & \text { if } f\left(u_{i, G+1}\right) \leq f\left(x_{i, G}\right), \\
x_{i, G}, & \text { otherwise. }\end{cases}
\end{aligned}
$$

As it is reviewed, back analysis based on the monitored displacement is useful when the displacement is significant due to excavation. On the other hand, the internal displacement of a segment-lined tunnel after the construction is trivial so that the application of conventional displacement-based back analysis is unsuitable. Thus, the conventional DEA using the monitored displacement is modified to consider the monitored stress information as well as the displacement to enhance the convergence in this study. The error function should be revised to accommodate the different observation variables.

\section{Numerical Model}

To perform back analysis, DEA was implemented using the FISH language embedded in the FLAC3D program (Itasca Consulting Group Inc. [11]) and the segmented lined tunnel was modeled to examine the performance of the established back analysis system. A segment-lined tunnel was idealized and the hydrostatic pressure was applied to the quartersymmetry geometry, as shown in Figure $4(\mathrm{a})\left(K_{0}=1\right)$.
The internal displacement monitoring points shown in Figure 4(b) were set in 5 positions at $15^{\circ}$ along the excavated tunnel periphery after the excavation. Figure 4(c) presents the segment-lined tunnel model. The internal displacement was monitored in the same manner along the lined tunnel periphery and stress acting on the segment lining was also monitored at three points.

The linear elastic and perfectly plastic Mohr-Coulomb model was assumed for weathered rock and its material properties are tabulated in Table 1: $2.1 \mathrm{GPa}$ elastic modulus, 0.2 Poisson's ratio, $1.1 \mathrm{MPa}$ cohesion, and $30^{\circ}$ friction angle (Song et al. [20]). The thickness of the segment lining was $20 \mathrm{~cm}$ and its elastic properties $(E=24 \mathrm{GPa}, \nu=0.18$, $\gamma_{\mathrm{t}}=25 \mathrm{kN} / \mathrm{m}^{3}$ ) were applied to the corresponding zone.

The DEA parameters should be defined for effective back analysis, such as mutant constant, crossover constant, number of population, and maximum number of iterations. The optimal parameters are searched and tabulated in Table 2, which provides the best convergence performance: 10 for the number of population, 0.8 for the mutant constant, 0.8 for the crossover constant, and 10 for the maximum generation number. Moreover, the three target variables of back analysis were selected and the ranges were defined as $0.0 \mathrm{GPa} \leq E \leq 1.0 \mathrm{GPa}, 0.0 \mathrm{MPa} \leq c \leq 0.8 \mathrm{MPa}, 25^{\circ} \leq \phi \leq 38^{\circ}$.

To examine the applicability of the proposed back analysis system for a segment-lined tunnel under high groundwater pressure conditions, the radius of the tunnel varies $3 \mathrm{~m}$ and $5 \mathrm{~m}$. Figure 5 shows the boundary and geometry of the three representative cases considered in this study. In particular, the monitored displacements were only observed and used for back analysis to confirm the validity of the established back analysis system based on DEA in FLAC3D. Therefore, the fixed hydrostatic geostress (i.e., $p_{0}=10 \mathrm{MPa}$ and $20 \mathrm{MPa}$ ) was applied at the boundary, as shown in Figure 5.

In addition, DEA was extended and modified to accommodate the monitored stress information. Figure 6 presents the representative numerical model for back analysis based on the modified DEA using stress and displacement for segment-lined tunnel. To examine the performance of the modified DEA for segment-lined tunnel, $1 \mathrm{MPa}$ of additional isotropic hydrostatic pressure was applied around the boundary to generate a small amount of additional displacement, as shown in Figure 6(b). In this study, 9 analysis cases are summarized in Table 3 considering the observation parameters, thickness of segment lining, radius of tunnel, and hydrostatic pressure.

\section{Analysis and Result}

4.1. Back Analysis Using Displacement. Figure 7 presents a flowchart of back analysis based on DEA using monitored radial displacement. First, the numerical models for each case should be established with predefined boundary conditions using FLAC3D. In addition, radial displacements in the $x$-direction $(D x)$ and $z$-direction $(D z)$ were monitored at the five predefined positions. The displacement obtained from this initial analysis can be considered to be true observations with known target variables. The back analysis 


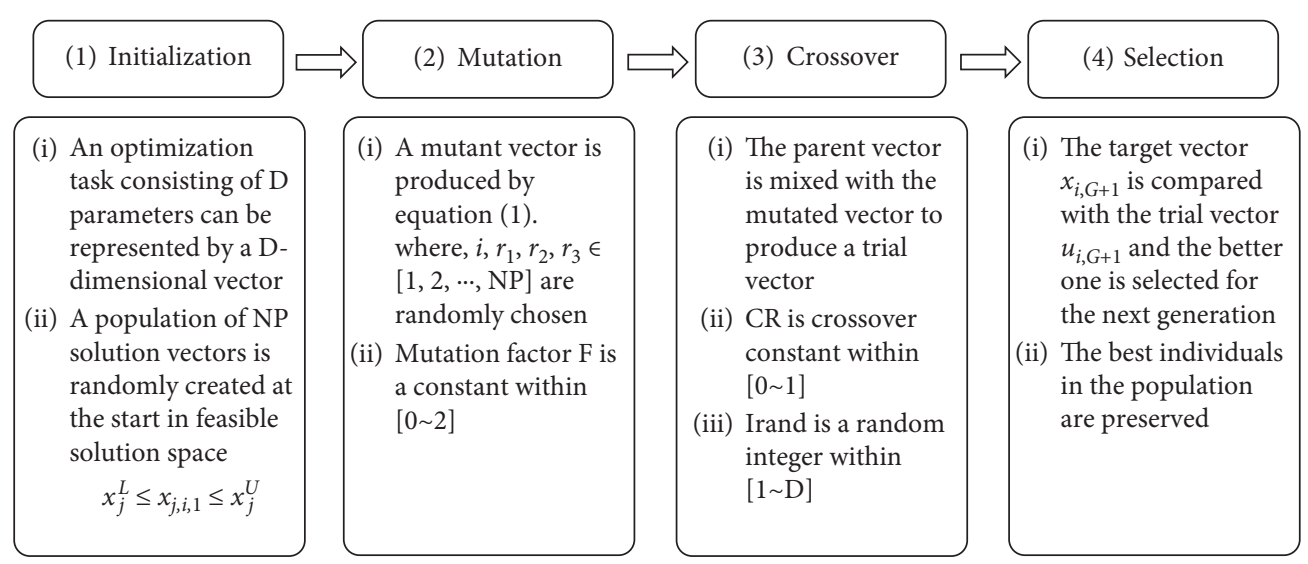

Figure 2: Main stages of DEA (Storn and Price [19]).

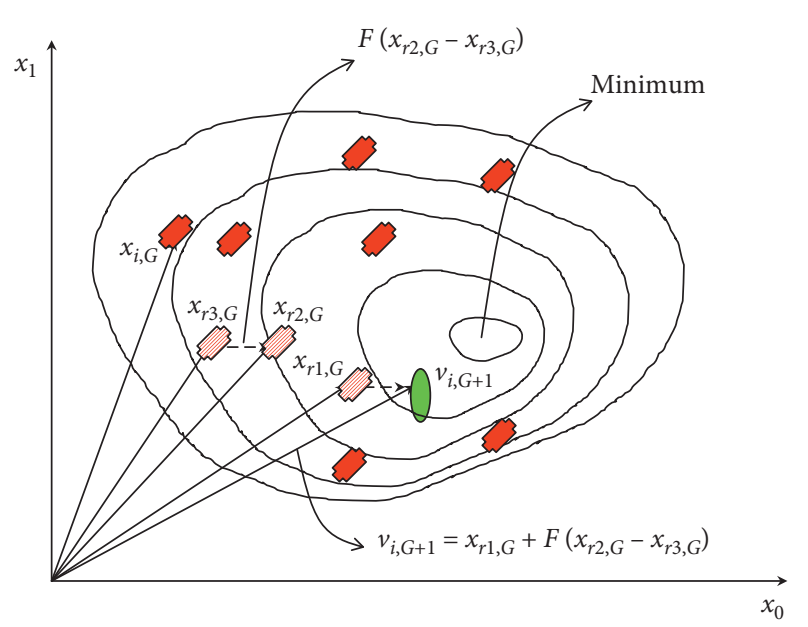

Random vector of G-generation
Selection for mutation vector
New generation vector

Figure 3: DEA process in two-dimensional space (Storn and Price [19]).

should be performed by implementing DEA to find the target variables that satisfy the true displacement observations.

The iterative procedure of DEA is composed of initialization, mutation, crossover, selection, and evaluation ( $\mathrm{Su}$ et al. [8]). The iterative calculation will be finished if the error function of the trial displacement vector falls within the maximum permissible error, or if a generation number reaches the maximum number. Equation (4) defines the error function with three displacement components for an evaluation of trial displacement vector with respect to the true observation in DEA. The error function used in this study is the sum of the difference between the true displacement observations $\left(\mathrm{d} x_{i}, \mathrm{~d} y_{i}, \mathrm{~d} z_{i}\right)$ obtained from the initial analysis with known variables and the trial displacement vectors $\left(D x_{i}, D y_{i}, D z_{i}\right)$ with the trial target variables. $i$ indicates the position of the monitoring point and $n$ denotes the total number of the monitoring points.

$$
f=\sum_{i=1}^{n} \sqrt{\left[\left(\mathrm{d} x_{i}-D x_{i}\right)^{2}+\left(\mathrm{d} y_{i}-D y_{i}\right)^{2}+\left(\mathrm{d} z_{i}-D z_{i}\right)^{2}\right]} .
$$

Target vectors with the minimum error are considered to be the optimal solution. Otherwise, the iteration will be resumed at the mutation stage to obtain a trial target vector of the next generation. The newly generated trial target vectors are updated as material properties and the numerical analysis is performed to obtain the trial displacement vectors with respect to the trial target vectors.

In this study, three target variables were selected for back analysis: elastic modulus, cohesion, and friction angle of ground. The back analysis was performed with DEA using two internal displacement components $(D x$ and $D z)$ under various boundary conditions (Case A). Table 4 lists the error of the trial target variables and the mean of the target variables errors with respect to the true observations for Case A. In general, the error of the friction angle is smaller than that of the other target variables. In addition, Case A-3, where the monitored displacement is relatively larger than the other cases, shows the smallest average error of 3.6\%. Overall, the average error of cases $\mathrm{A}$ is less than $5 \%$, which means that the back analysis can be performed readily with displacement information when the displacement is significant. This confirms that the established back analysis system is valid.

Furthermore, the applicability of back analysis based on DEA using the displacement was examined with a segmentlined tunnel. The internal displacement is trivial after the installation of segment lining. Therefore, $1 \mathrm{MPa}$ of additional hydrostatic pressure is applied to the boundary to develop a small amount of internal displacement. The back analysis was performed based on the flowchart presented in Figure 7 , and the results are listed in Table 5. Although the error of the friction angle was the smallest among the target variables, the error was greater than $5.7 \%$. The error of the elastic modulus was greater than $42 \%$ and cohesion showed the worst error of $50 \%$. In general, the average error of the target variable was greater than $32 \%$ when the back analysis was performed with small displacement only. In all cases, the evolution calculation was finalized due to the maximum iteration criteria, which implies poor convergence. In 


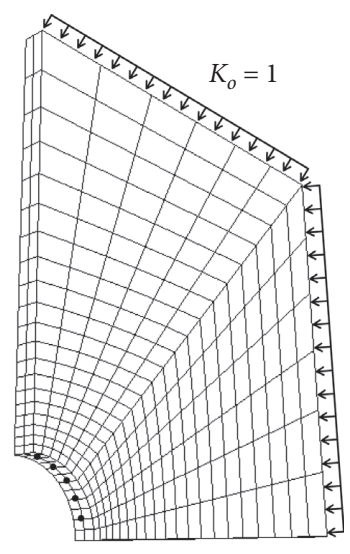

(a)

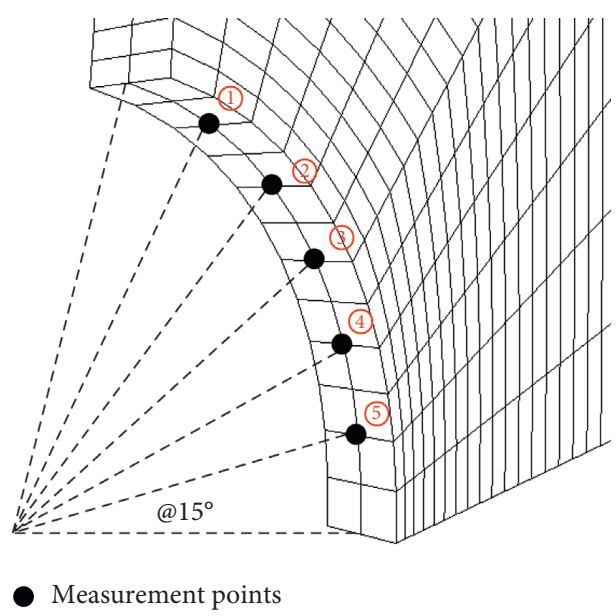

(b)

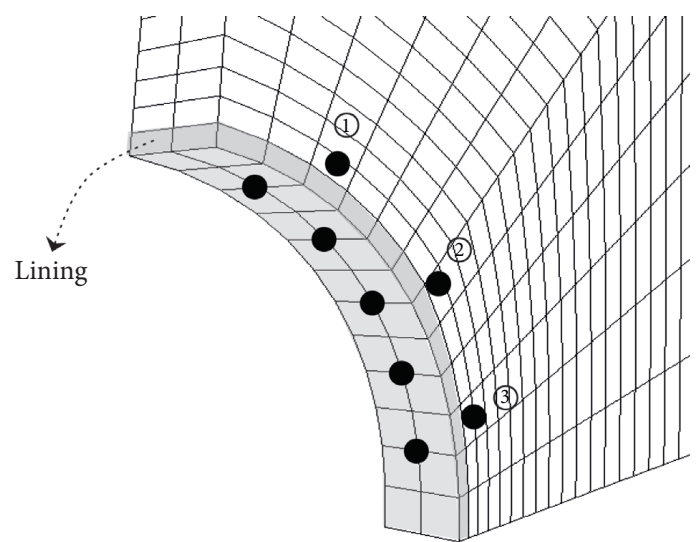

Measurement points

(c)

FIgURE 4: Construction of a numerical model and monitoring of displacement and stress. (a) Quarter-symmetry numerical model. (b) Displacement monitoring along the excavated tunnel periphery. (c) Stress and displacement monitoring along the lined tunnel periphery.

TABLE 1: Material properties used in the numerical study.

\begin{tabular}{lcc}
\hline & Lining & Ground (weathered rock) \\
\hline Elastic modulus, $E$ & $24 \mathrm{GPa}$ & $0.5 \mathrm{GPa}$ \\
Cohesion, $c$ & - & $0.25 \mathrm{MPa}$ \\
Friction angle, $\phi$ & - & $35^{\circ}$ \\
Poisson's ratio, $v$ & 0.18 & 0.3 \\
Model & Elastic & Mohr-coulomb \\
Bulk modulus, $K$ & $E / 3 \cdot(1-2 \nu)$ & \\
Shear modulus, $G$ & $E / 2 \cdot(1+\nu)$ & \\
\hline
\end{tabular}

TABLE 2: DEA properties for back analysis.

\begin{tabular}{lc}
\hline Parameters & Value \\
\hline & $0.0 \mathrm{GPa} \leq E \leq 1.0 \mathrm{GPa}$, \\
Range of target variables & $0.0 \mathrm{MPa} \leq c \leq 0.8 \mathrm{MPa}$, \\
& $25^{\circ} \leq \phi \leq 38^{\circ}$ \\
\hline Mutant constant, F & $0.8(0 \sim 2)$ \\
Crossover constant, CR & $0.8(0 \sim 1)$ \\
Number of population, NP & 10 \\
Maximum generation number & 10 \\
\hline
\end{tabular}

summary, the conventional displacement-based back analysis is unsuitable for a segment-lined tunnel that shows small amount of displacement.

4.2. Back Analysis Using Displacement and Stress. As found in the previous chapter, the performance of displacementbased back analysis using DEA is limited due to the convergence problem and high error. Therefore, the conventional displacement-based back analysis scheme should be improved for an analysis of a segment-lined tunnel. In this study, stress acting on the segment lining is also considered as evolutionary entities as well as internal displacement.

When the internal displacement is minor, the trial displacement vector does not respond sensitively to a change in the trial target variable. In other words, the convergence conditions can be satisfied due to the small difference between the trial displacement vector and true observation, but the target variables are often far out-positioned from the acceptable target range. Therefore, the incremental error function was selected in this study to represent the convergence efficiency of the calculation result, as shown in equation (5) for displacement and equation (6) for stress. The convergence efficiency was not influenced by the amplitude of displacement and stress because the increment is normalized and nondimensionalized. Moreover, an excellent convergence efficiency was obtained when the incremental error function was used in the back analysis with a minor displacement. Two different error functions for stress and displacement can be executed simultaneously. The incremental error function of DEA for displacement can be defined as a sum of the difference between the true displacement observation $\left(\mathrm{d} x_{i}, \mathrm{~d} y_{i}, \mathrm{~d} z_{i}\right)$ and the trial displacement vector $\left(D x_{i}, D y_{i}, D z_{i}\right)$, as shown in equation (5). The incremental error function of DEA for stress can be obtained from the true stress observation $\left(\sigma_{1 R}, \sigma_{2 R}, \sigma_{3 R}\right)$ and the trial stress vector $\left(\sigma_{1}, \sigma_{2}, \sigma_{3}\right)$, as shown in equation (6).

$$
f=\sum_{i=1}^{n} \sqrt{\left[\left(\frac{\mathrm{d} x_{i}-\mathrm{D} x_{i}}{\mathrm{~d} x_{i}}\right)^{2}+\left(\frac{\mathrm{d} y_{i}-\mathrm{D} y_{i}}{\mathrm{~d} y_{i}}\right)^{2}+\left(\frac{\mathrm{d} z_{i}-\mathrm{D} z_{i}}{\mathrm{~d} z_{i}}\right)^{2}\right]},
$$

$$
f=\sum_{i=1}^{n} \sqrt{\left[\left(\frac{\sigma_{1 R}-\sigma_{1}}{\sigma_{1 R}}\right)^{2}+\left(\frac{\sigma_{2 R}-\sigma_{2}}{\sigma_{2 R}}\right)^{2}+\left(\frac{\sigma_{3 R}-\sigma_{3}}{\sigma_{3 R}}\right)^{2}\right]} .
$$

Figure 7 shows the back analysis process using modified DEA, which can accommodate the stress and displacement components. Similarly, in Case B, an additional hydrostatic pressure was applied along the boundary to develop a small amount of additional internal displacement after the simulation of excavation and lining installation in Case C. In 
$10 \mathrm{MPa}$

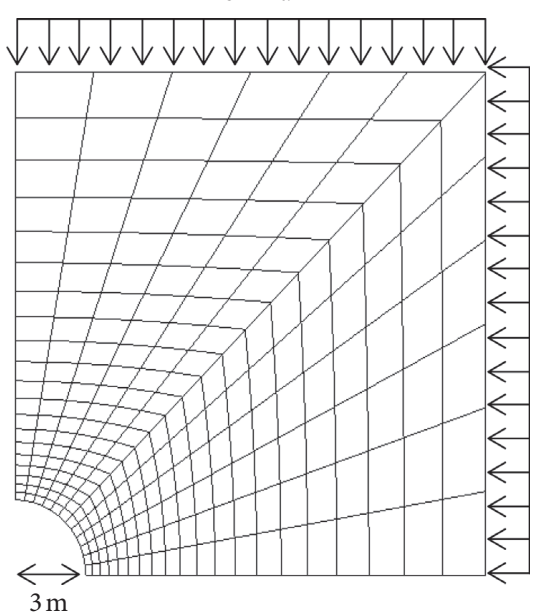

(a)
$10 \mathrm{MPa}$

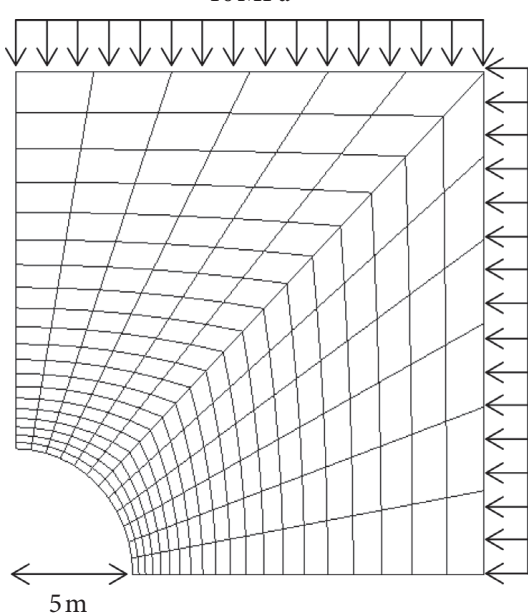

(b)

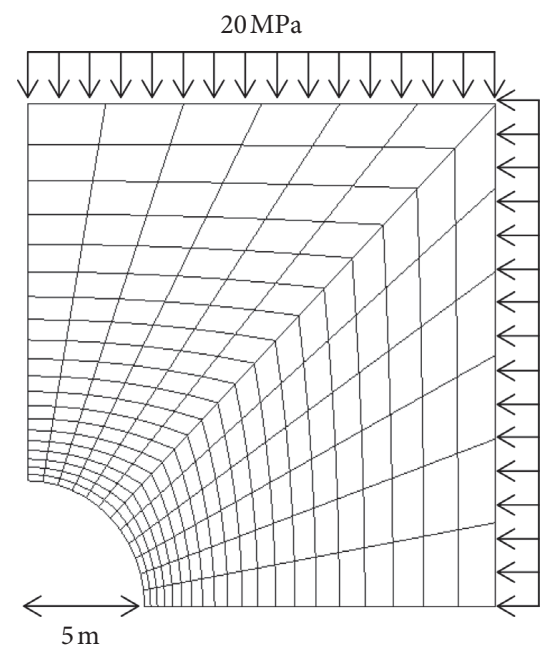

(c)

Figure 5: Numerical model cases for the DEA calculations (displacement-based).

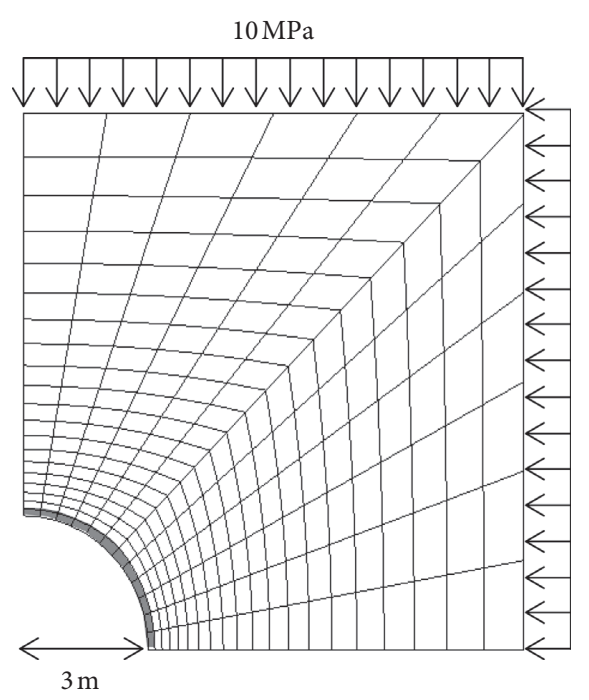

(a)

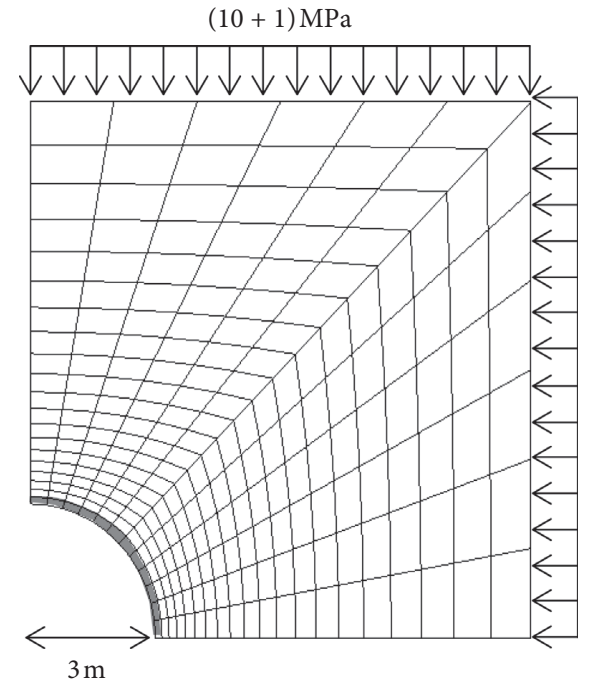

(b)

FIgUre 6: Numerical model for back analysis based on the modified DEA using stress and displacement. (a) Segment-lined tunnel under hydrostatic pressure. (b) Segment-lined tunnel with $1 \mathrm{MPa}$ of additional isotropic hydrostatic pressure.

Table 3: Summary of analysis cases.

\begin{tabular}{|c|c|c|c|c|}
\hline Cases & Observation parameters & Thickness of segment lining & Radius of tunnel & Hydrostatic pressure \\
\hline A-1 & \multirow{3}{*}{ Displacement } & - & $3 \mathrm{~m}$ & $10 \mathrm{MPa}$ \\
\hline A-2 & & - & $5 \mathrm{~m}$ & $10 \mathrm{MPa}$ \\
\hline A-3 & & - & $5 \mathrm{~m}$ & $20 \mathrm{MPa}$ \\
\hline B-1 & \multirow{3}{*}{ Displacement } & $0.2 \mathrm{~m}$ & $3 \mathrm{~m}$ & $11 \mathrm{MPa}$ \\
\hline B-2 & & $0.2 \mathrm{~m}$ & $5 \mathrm{~m}$ & $11 \mathrm{MPa}$ \\
\hline B-3 & & $0.2 \mathrm{~m}$ & $5 \mathrm{~m}$ & $21 \mathrm{MPa}$ \\
\hline $\mathrm{C}-1$ & \multirow{3}{*}{ Displacement and stress } & $0.2 \mathrm{~m}$ & $3 \mathrm{~m}$ & $11 \mathrm{MPa}$ \\
\hline $\mathrm{C}-2$ & & $0.2 \mathrm{~m}$ & $5 \mathrm{~m}$ & $11 \mathrm{MPa}$ \\
\hline $\mathrm{C}-3$ & & $0.2 \mathrm{~m}$ & $5 \mathrm{~m}$ & $21 \mathrm{MPa}$ \\
\hline
\end{tabular}




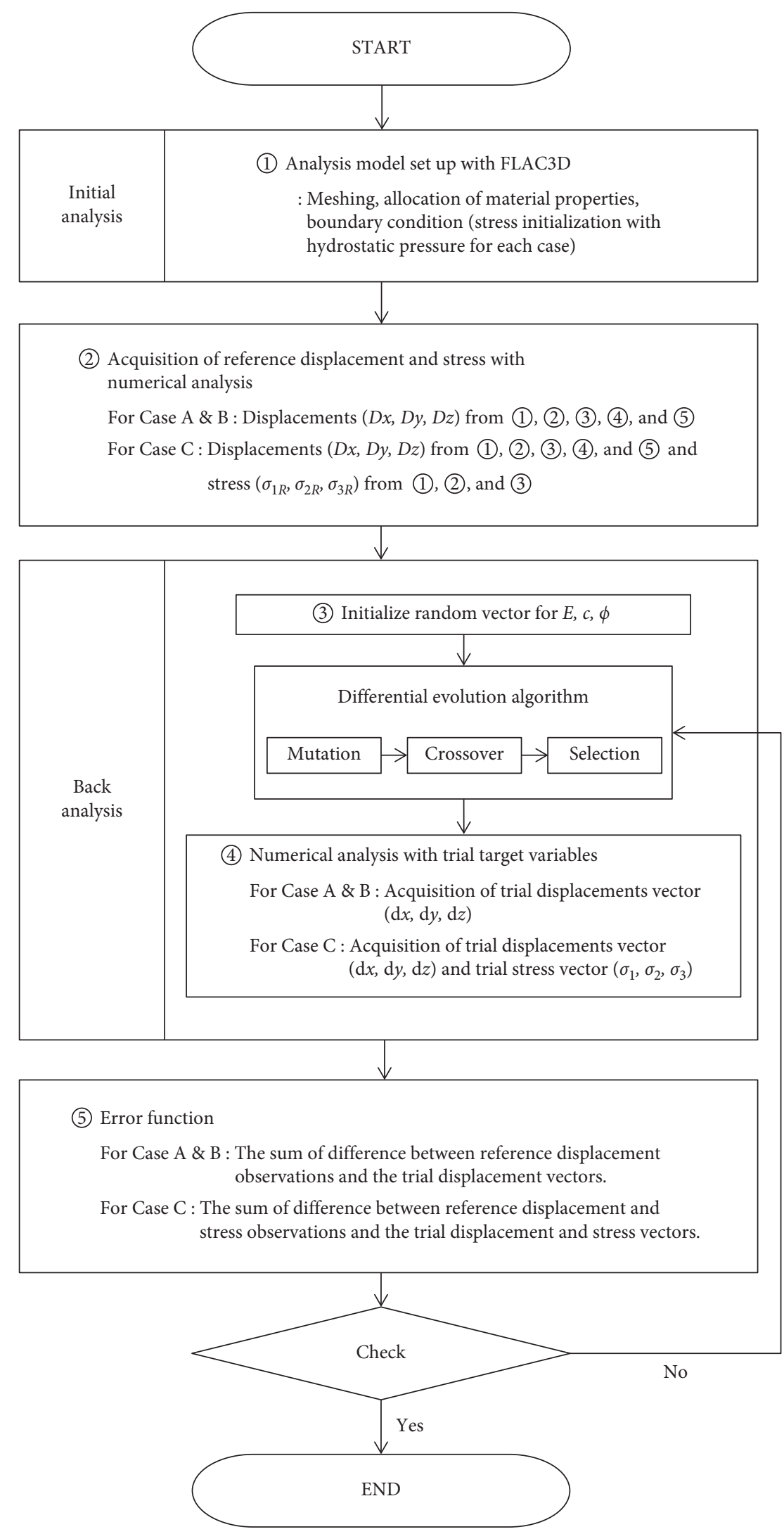

Figure 7: Flowcharts of back analysis based on DEA using monitored radial displacement and stress. 
TABLE 4: Results of back analysis using the displacement induced by excavation.

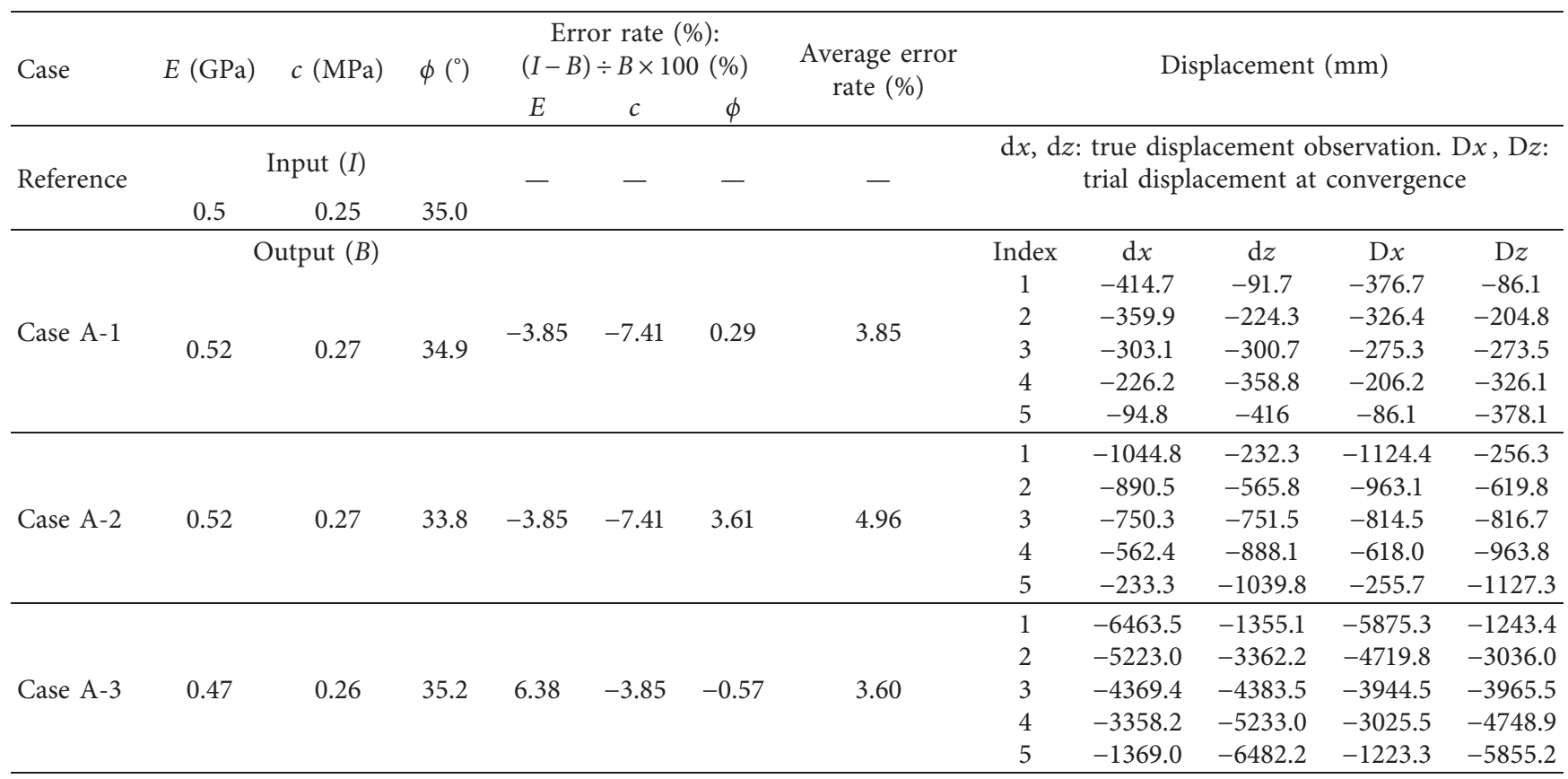

TABLE 5: Results of back analysis on a segment-lined tunnel using displacement.

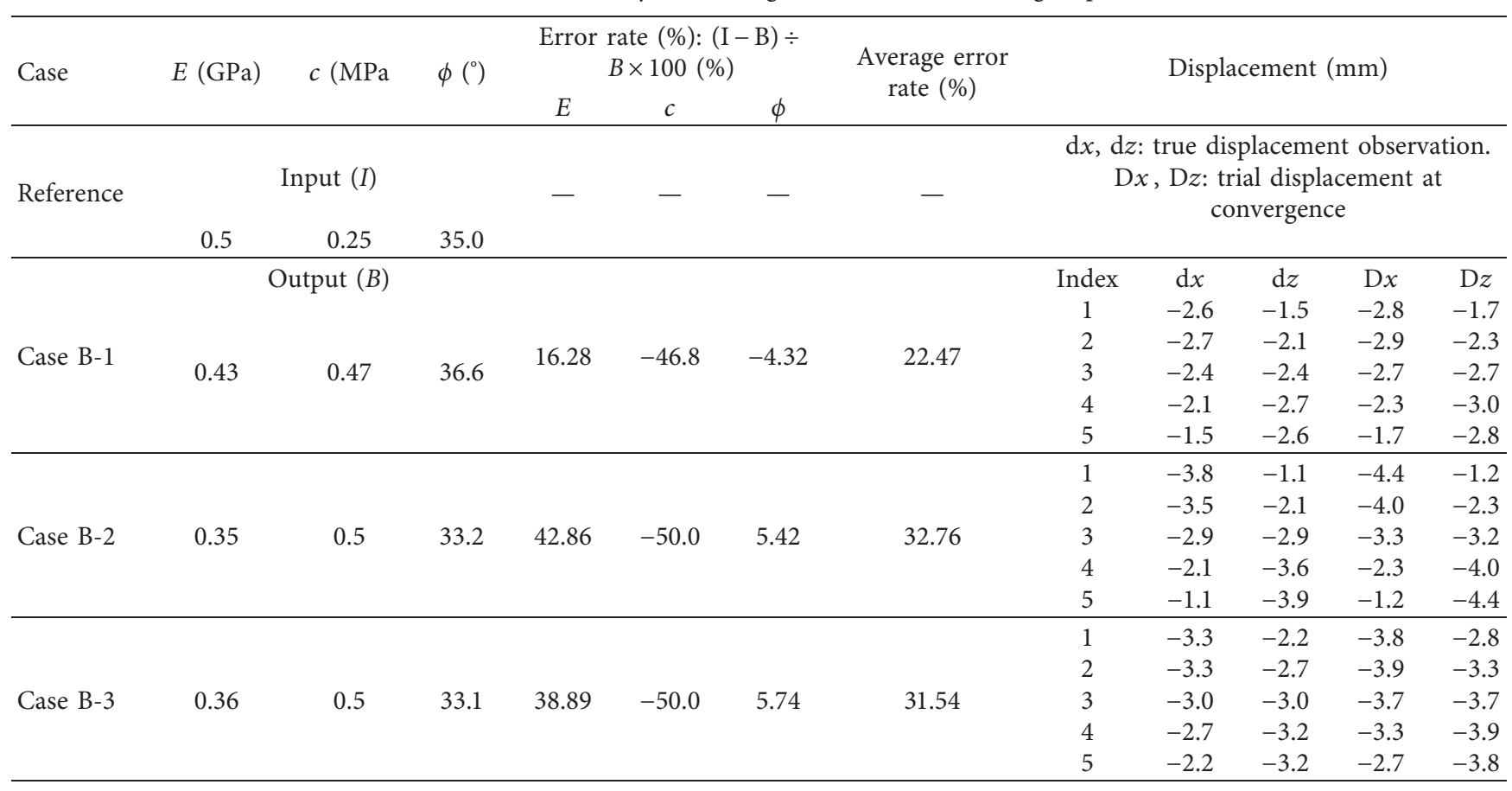

particular, the back analysis was performed with DEA, which was improved to accommodate four target variables (two displacements and two stresses) because the numerical study was performed under plane-strain conditions. Furthermore, the two different error functions for stress and displacement were implemented to evaluate the suitability of the target variables.

Table 6 lists the back analysis result on a segment-lined tunnel using stress and displacement. The error of the elastic modulus for a small-diameter tunnel was improved slightly. On the other hand, the error of the cohesion was improved significantly with an increase in tunnel radius and a hydrostatic pressure below 9\%. Although the error of cohesion is not sensitive to the boundary conditions, it is improved greatly compared to the result obtained from Table 5 with the displacement-based back analysis. The estimated average error ranged approximately 6-8\%, which suggests that the error can be reduced dramatically with the 
TABLE 6: Results of back analysis on a segment-lined tunnel using stress and displacement.

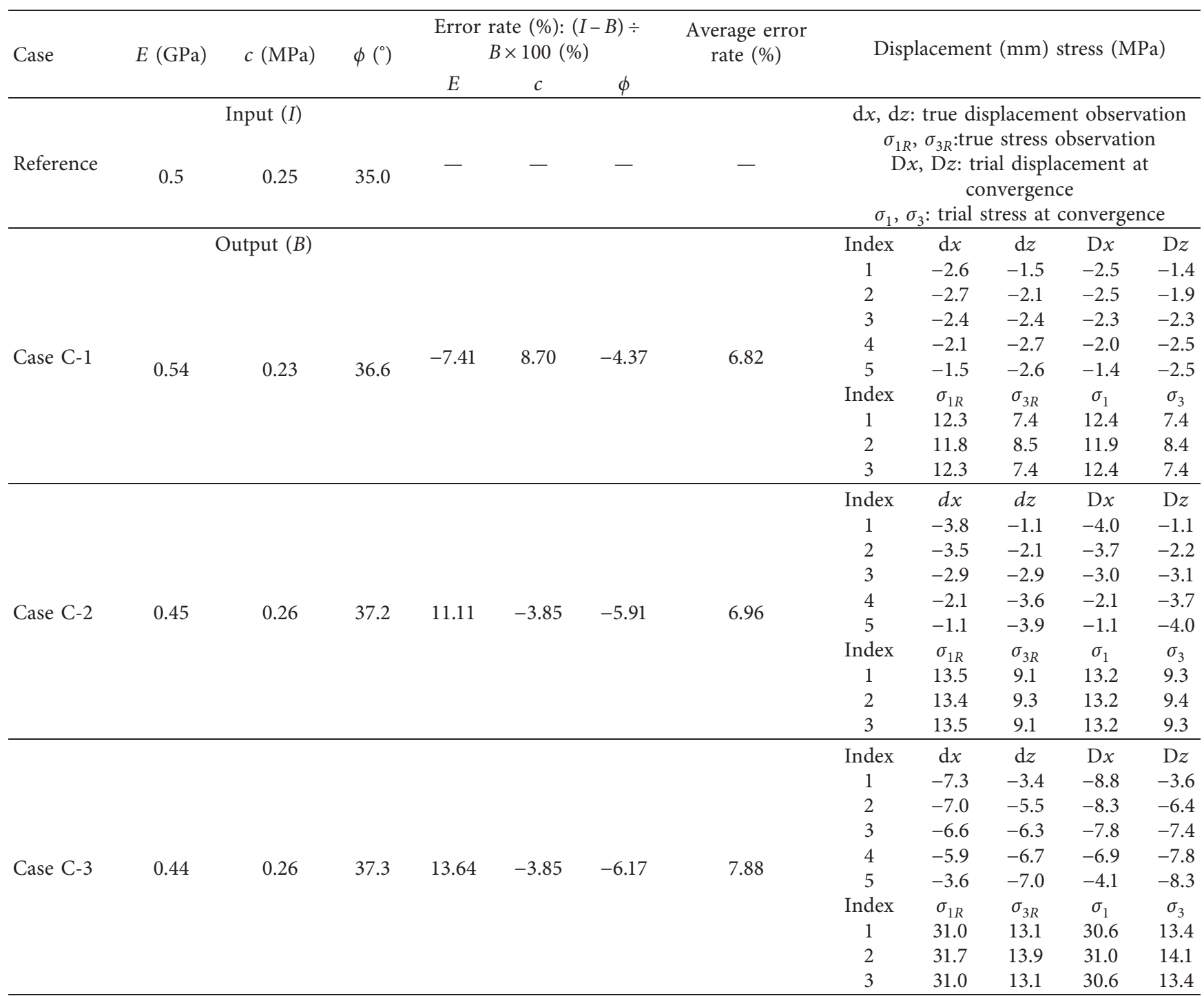

proposed back analysis algorithm using both the stress and displacement with respect to the conventional method using displacement.

\section{Conclusions}

Because the length of segment-lined tunnels driven by a mechanized tunneling method is increasing, a precise evaluation of the tunnel behavior after construction is essential. On the other hand, because the internal displacement of a segment-lined tunnel is trivial, it is difficult to analyze the stability of a segment-lined tunnel using the conventional method. In this study, the back analysis method on a segment-lined tunnel based on the differential evolution algorithm using stress and displacement was proposed. The back analysis based on the differential evolution algorithm using the stress and displacement was established and performed using the finite difference code, FLAC3D, and built-in FISH language. Detailed flowcharts of back analysis based on DEA using both monitored displacement stresses were also suggested. As a preliminary study, the ground properties were selected as the target variables for back analysis.

The back analysis based on the monitored displacement is useful when the displacement is significant due to excavation because the average error of Case A was less than $5 \%$. The average error of Case B was greater than $32 \%$ and the evolutionary calculation is finalized due to the maximum iteration criteria, which implies the poor convergence. Therefore, the conventional displacementbased back analysis is unsuitable for a segment-lined tunnel after construction because of its trivial internal displacement.

The conventional DEA was modified to consider the monitored stress and displacement information to enhance the convergence. The error functions are also revised to accommodate the various observation variables. The average error obtained from the proposed back analysis algorithm using both stress and displacement ranged within 
approximately $6-8 \%$. This suggests that the error can be significantly reduced with respect to the displacement-based conventional back analysis algorithm. This also confirms that the proposed back analysis algorithm is suitable for a segment-lined tunnel.

\section{Data Availability}

The data used to support the findings of this study are included within the article.

\section{Conflicts of Interest}

The authors declare that they have no conflicts of interest.

\section{Acknowledgments}

This research was supported by the Inha University Research Grant.

\section{References}

[1] G. Zhang, J. Tan, L. Zhang, and Y. Xiang, "Linear regression analysis for factors influencing displacement of high-filled embankment slopes," Geomechanics and Engineering, vol. 8, no. 4, pp. 511-521, 2015.

[2] W. Zhou, S.-L. Li, G. Ma, X.-L. Chang, Y.-G. Cheng, and $\mathrm{X}$. Ma, "Assessment of the crest cracks of the pubugou rockfill dam based on parameters back analysis," Geomechanics and Engineering, vol. 11, no. 4, pp. 571-585, 2016.

[3] W. Gao and T. Y. He, "Displacement prediction in geotechnical engineering based on evolutionary neural network," Geomechanics and Engineering, vol. 13, no. 5, pp. 845-860, 2017.

[4] M. Hisataki and T. Ito, "Back analysis for tunnels by optimization method," in Proceedings of the International Conference on Numerical Methods in Geomechanics, pp. 1301-1307, Nayoga, Japan, April 1985.

[5] G. Gioda and S. Sakurai, "Back analysis procedures for the interpretation of field measurements in geomechanics," International Journal for Numerical and Analytical Methods in Geomechanics, vol. 11, no. 6, pp. 555-583, 1987.

[6] J.-S. An and K.-I. Song, "Back analysis of an operating subsea tunnel considering the degradation of ground and concrete lining," Marine Georesources \& Geotechnology, vol. 37, no. 4, pp. 517-523, 2019.

[7] S. Vardakos, Back-analysis methods for optimal tunnel design, Virginia Polytechnic Institute and State University, Blacksburg, VA, USA, Ph.D. Dissertation, 2007.

[8] G. S. Su, X. F. Zhang, G. Q. Chen, and X. Y. Fu, "Identification of structure and parameters of rheological constitutive model for rocks using differential evolution algorithm," Journal of Central South University of Technology, vol. 15, no. 1, pp. 25-28, 2008.

[9] J. H. Lee and S. Akutagawa, "Quick prediction of tunnel displacements using artificial neural network and field measurement results," International Journal of the JCRM, vol. 5, no. 2, pp. 53-62, 2009.

[10] S. Vardakos, M. Gutierrez, and C. Xia, "Parameter identification in numerical modeling of tunneling using the differential evolution genetic algorithm (DEGA)," Tunnelling and Underground Space Technology, vol. 28, pp. 109-123, 2012.
[11] Itasca Consulting Group Inc., FLAC3D-Fast Lagrangian Analysis of Continua in 3 Dimensions, Itasca Consulting Group Inc., Minneapolis, MN, USA, 2012.

[12] H.-S. Hwang, "Function optimization and event clustering by adaptive differential evolution," Journal of Korean Institute of Intelligent Systems, vol. 12, no. 5, pp. 451-461, 2002.

[13] J. Vesterstrøm and R. Thomsen, "A comparative study of differential evolution, particle swarm optimization, and evolutionary algorithms on numerical benchmark problems," in Proceedings of the Congress on Evolutionary Computation (CEC'04), vol. 2, pp. 1980-1987, Portland, OR, USA, June 2004.

[14] B. Růžek and M. Kvasnička, "Differential evolution algorithm in the earthquake hypocenter location," Pure and Applied Geophysics, vol. 158, no. 4, pp. 667-693, 2001.

[15] P. M. Reed and S. Yamaguchi, "Simplifying the parameterization of real-coded evolutionary algorithms," in Proceedings of the Critical Transitions in Water and Environmental Resources Management, pp. 1-9, Salt Lake City, UT, USA, June 2004.

[16] J. Decker and M. Mauldon, "Determining size and shape of fractures from trace data using a differential evolution algorithm," in Proceedings of the 41st US Symposium on Rock Mechanics (USRMS), Golden, CL, USA, June 2006.

[17] S. M. Seyedpoor, E. Norouzi, and S. Ghasemi, "Structural damage detection using a multi-stage improved differential evolution expealgorithm," Smart Structures and Systems, vol. 21, no. 2, pp. 235-248, 2018.

[18] S. M. Seyedpoor and M. Montazer, "A two-stage damage detection method for truss structures using a modal residual vector based indicator and differential evolution algorithm," Smart Structures and Systems, vol. 17, no. 2, pp. 347-361, 2016.

[19] R. Storn and K. Price, "Differential evolution-a simple and efficient heuristic for global optimization over continuous spaces," Journal of Global Optimization, vol. 11, no. 4, pp. 341-359, 1997.

[20] K.-I. Song, G.-C. Cho, and S.-W. Lee, "Effects of spatially variable weathered rock properties on tunnel behavior," Probabilistic Engineering Mechanics, vol. 26, no. 3, pp. 413426, 2011. 